Case report

\title{
Rare complication of diagnostic coronary angiography: Perforation is
}

\author{
Hakan Güneş ${ }^{\text {a,* }}$, Ömer Şahin ${ }^{\text {a }}$, Hasan Ata Bolayır ${ }^{\text {a }}$, Mustafa Adem Tatlısu ${ }^{\text {a }}$, Tarık Kıvrak ${ }^{\text {a }}$, Ilgın Karaca ${ }^{\text {a,b }}$ \\ a Sivas State Hospital, Cardiology Department, Sivas, Turkey \\ b Firat University, Cardiology Department, Elazı̆̆, Turkey
}

\section{A R T I C L E I N F O}

\section{Article history:}

Received 7 November 2016

Received in revised form 29 January 2017

Accepted 30 January 2017

Available online 3 February 2017

\section{Introduction}

Diagnostic coronary angiography $(\mathrm{CA})$ and percutaneous coronary interventions ( $\mathrm{PCI}$ ) are being performed in increasing numbers. However, several complications may also be seen in daily practice. Most are vascular access site complications. Acute myocardial infarction, coronary artery perforation, stroke, and death are rare complications, with an incidence of around $1 \%{ }^{1}$ The coronary artery perforation is usually seen in $\mathrm{PCl}$, and we present a case of coronary artery perforation of the three major branches of the right coronary artery.

\section{Case}

The patient, a 45-year-old female renal transplant recipient, presented with chest pain accompanied by shortness of breath, which is precipitated by exertion, and relieved by rest. She did not have any symptoms of congestive heart failure. Her family history was significant for hypertension and hyperlipidemia. She had been attending hemodialysis three-times a week for 8 years for chronic renal failure. She had been undergoing hemodialysis through a temporary double lumen subclavian catheter for 1 week due to the nonfunctioning dialysis fistula. The electrocardiogram (ECG) revealed sinus rhythm with T-wave inversion

\footnotetext{
is Peer review under responsibility of The Society of Cardiovascular Academy

* Corresponding author.

E-mail address: drhakangunes83@hotmail.com (H. Güneș).
}

in leads II, III, and aVF. The transthoracic echocardiography (TTE) showed an ejection fraction of 55\%, normal cardiac chamber dimensions, and impaired inferior wall motion, which might be associated with coronary fistulae. TTE also showed minimal tricuspid, and mitral regurgitation. The patient was advised to undergo CA, based on symptoms and findings consistent with the coronary artery disease. The CA was performed using the standard femoral approach with a 6-Fr diagnostic catheter. The CA revealed mild plaques in the left anterior descending artery and left circumflex artery (Figs. 1-2). In the left anterior oblique view, fistulas were seen between proximal right coronary artery (RCA) and right atrium. In right anterior oblique view, the small localized contrast staining was seen, which was consistent with the perforation of distal three branches of RCA (Figs. 3-5). The ECG was obtained due to the new-onset chest pain. It showed no change from previous ECG. The cardiac panel showed that CK-MB levels were minimally elevated, and troponin-I levels were normal. The TTE revealed a small pericardial effusion without tamponade. She was admitted to the coronary intensive care unit for follow-up monitorization. The pericardial effusion did not change during a 48 -hour follow-up. The patient was advised to undergo percutaneous coil embolization of fistulas of RCA by our heart team. She was referred to the tertiary cardiovascular center.

\section{Discussion}

The complications of CA can be divided into three groups: major, minor, and contrast-associated. Minor complications include vascular access site bleeding, bruising, and infections. Major complications include ventricular fibrillation, acute myocardial infarction, stroke, aortic 


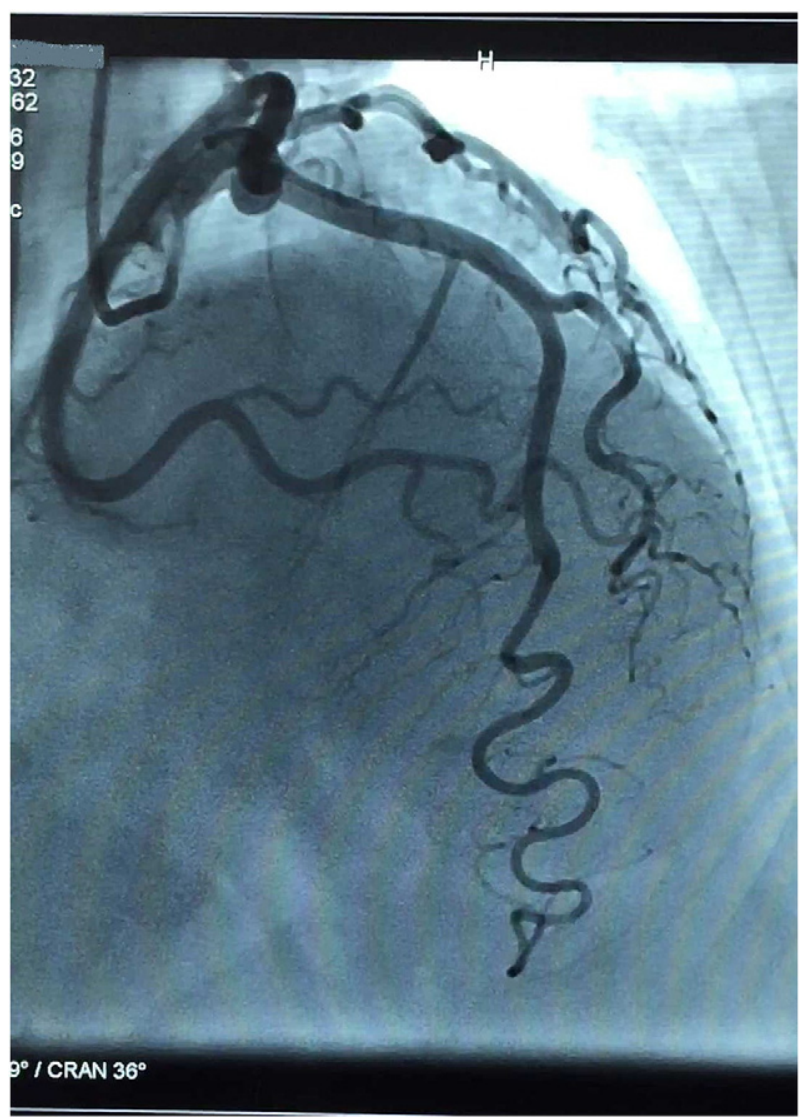

Fig. 1. Right Anterior Oblique views (RAO) with cranial angulation. Normal LAD.

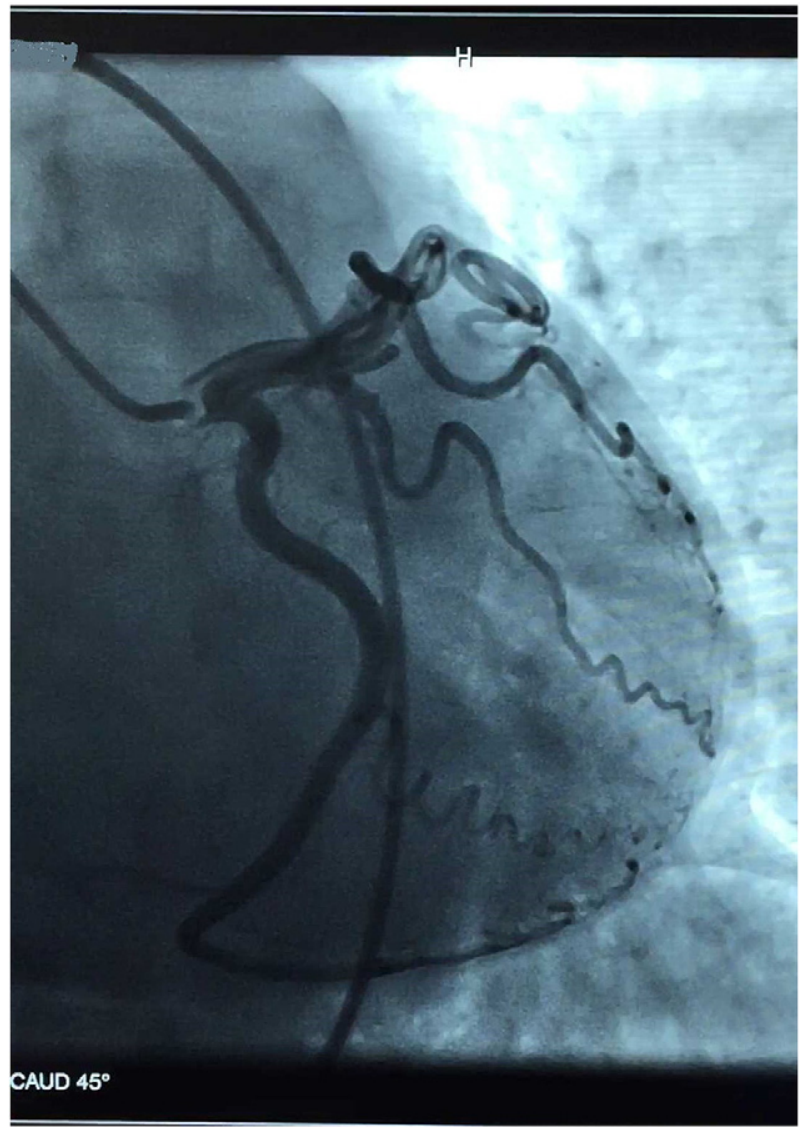

Fig. 2. Right Anterior Oblique views (RAO) with caudal angulation. Normal CX.

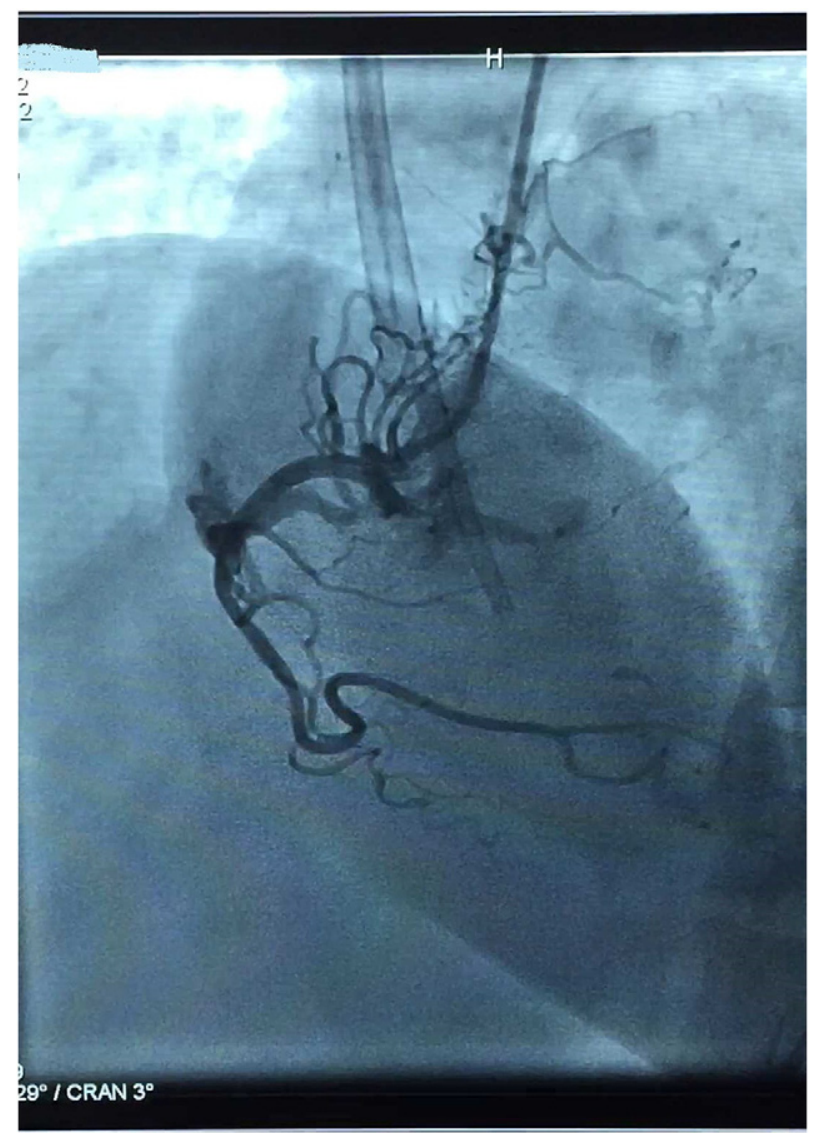

Fig. 3. Left Anterior Oblique views (LAO) with cranial angulation. Fistulas between proximal right coronary artery and right atrium.

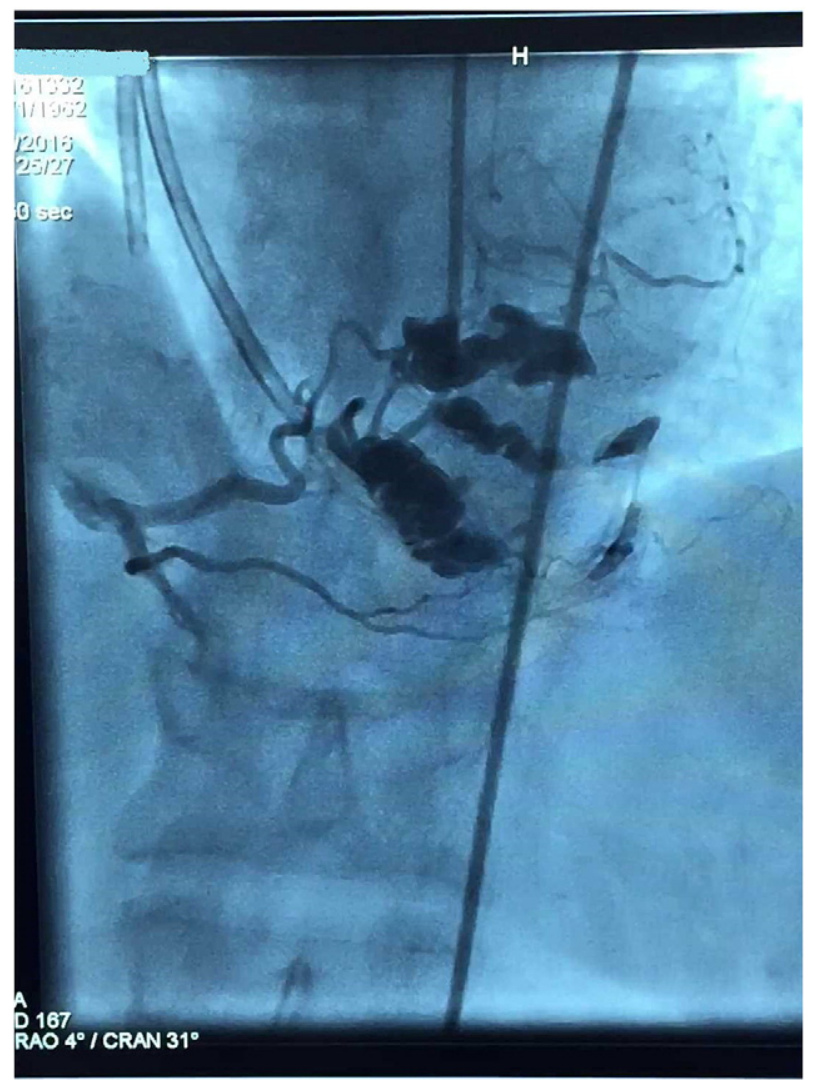

Fig. 4. Left Anterior Oblique views (LAO) with cranial angulation. Perforation of distal parts of proximal side branches of RCA 


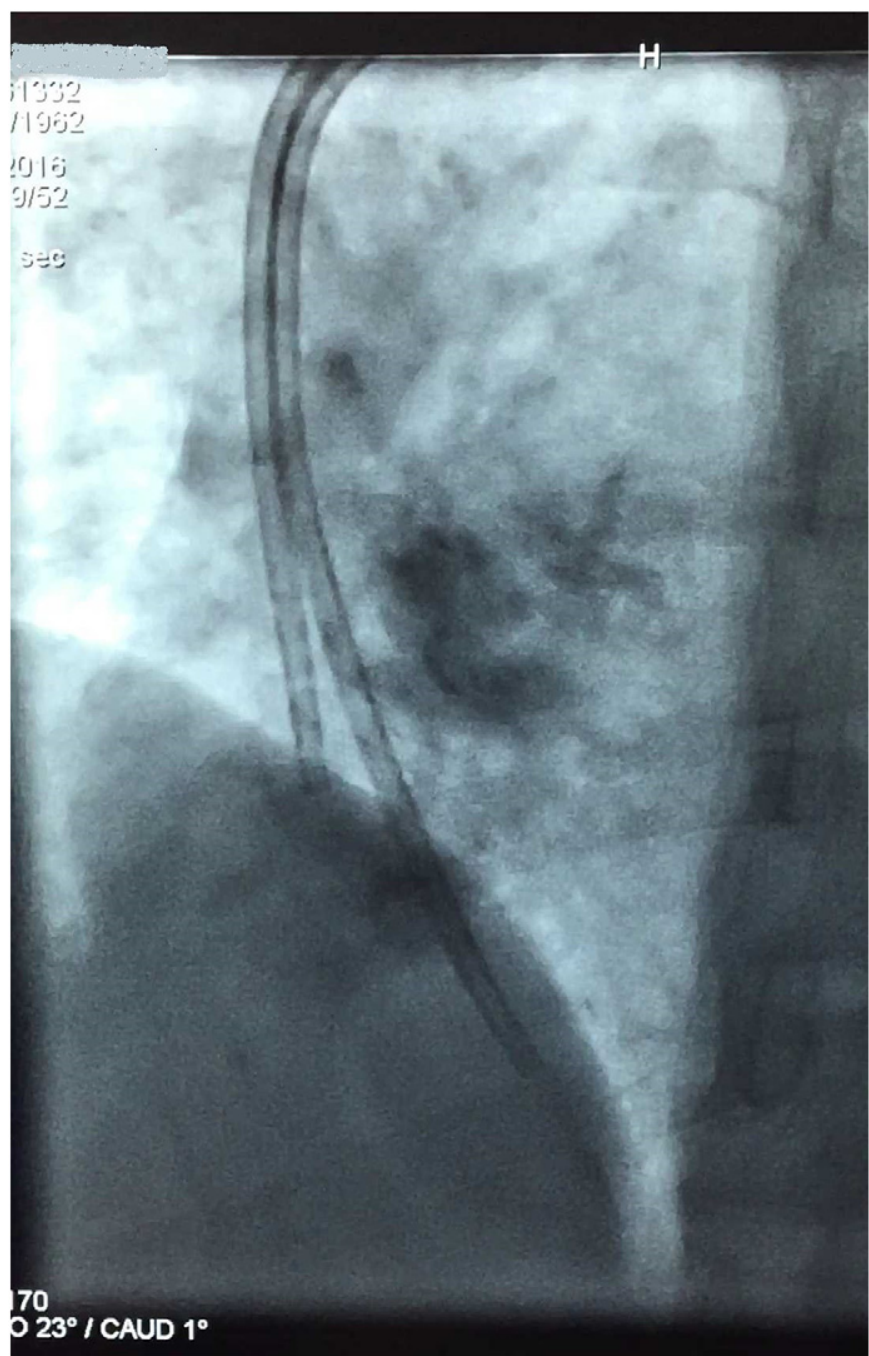

Fig. 5. Left Anterior Oblique views (LAO) with cranial angulation. The small localized contrast staining.

dissection, ventricular perforation, and coronary artery perforation. ${ }^{2-3}$ The coronary artery perforation is a complication of $\mathrm{PCI}$ rather than CA. The incidence of coronary perforation ranges from $0.29 \%$ to $0.59 \%{ }^{4-6}$ The risk factors for coronary perforation include age, gender (female), chronic kidney disease, and non-ST segment elevation myocardial infarction. ${ }^{6}$ Ellis et al. ${ }^{7}$ classified coronary perforation into three types: Type I (extraluminal crater), II (myocardial or pericardial blushing), and III (contrast streaming or cavity spilling). The coronary perforation in diagnostic CA is very rare and has been reported for the first time by Scöbel et al. ${ }^{8}$ The selective cannulation of the conus branch of RCA was presented in their case report. The $5 \mathrm{~F}$ right Judkins $(4 \mathrm{~cm})$ diagnostic catheter was used to view RCA in their case. The patient was managed conservatively. The control CA showed no evidence of contrast extravasation. Timurkaynak et al. ${ }^{9}$ presented another coronary perforation caused by deep cannulation of proximal branches of RCA. The $7 \mathrm{~F}$ right Judkins $(4 \mathrm{~cm})$ guiding catheter was used in their case. They did not find any pericardial effusion, and the patient was managed conservatively. Duyuler et al. ${ }^{10}$ reported a perforation of the intermediate coronary artery due to deep cannulation with the diagnostic catheter. The patient developed acute pericarditis and was managed conservatively.

In our case, the type II perforation occurred due to the deep cannulation of proximal RCA. The patient was managed conservatively even though the lesion could be managed with covered stents. Since the amount of pericardial effusion did not increase. According to our current knowledge, the avoidance of deep cannulation of coronary artery and forceful contrast injection can prevent coronary artery perforation.

\section{References}

1. Ökçün B, Gürmen T. Complications and the management strategies of coronary angiography. Turkiye Klinikleri J Int Med Sci 2007;3(42):48-72.

2. O'Sullivan JJ, McDonald K, Crean PA, Walsh MJ, McCarthy C, Erwin RJ, Maurer BJ. Cardiac catheterization with 5 French catheters. Br Heart J 1990;64:211-213.

3. Brown RIG, MacDonald AC. Use of 5 French catheters for cardiac catheterizasyon and coronary anjiography: critical review. Catheter Cardiovasc Diagn 1987;13:214-2017.

4. Ellis SG, Vandormeal MG, Cowley MJ. Coronary morphologic and clinical determinants of procedural outcome with an anjioplasty for multivessel coronary disease. Implication for patient selection. Multivessel Anjiioplasty Prognosis Study Group 1990;82(4):1193-2002.

5. Fasseas P, Orford JL, Panetta CJ, et al. Incidence, correlates, management, and clinical outcome of coronary perforation: analysis of 16,298 procedures. Am Heart J 2004; 147:104-105.

6. Doll JA, Nikolsky E, Stone GW, et al. Outcomes of patients with coronary artery perforation complicating percutaneous coronary intervention and correlation with the type of adjunctive antithrombotic therapy: pooled analysis from REPLACE-2 ACUTY, and HORIZONS-AMI trials. J Interv Cardiol 2009;22:453-459.

7. Teis A, Fernandez-Nofrerias E, Rodriguez-Leor $\mathrm{O}$, et al. Coronary artery perforation by intracoronary guidewires: risk factors and clinical outcomes. Rev Esp Cardiol 2010;63: 730-734.

8. Scöbel WA, Voelker W, Karsch KR. Perforation of side branch of right coronary artery during selective coronary anjiography using 5 French Judkins catheters. Catheter Cardiovasc Diagn 1995;36:156-159.

9. Timurkaynak T, Ciftci H, Cemri M. Coronary artere perforation: rare complication of coronary anjiogrphy. Acta Cardiol 2001;56(5):323-325.

10. Duyuler S, Bayır PT, Korkmaz A, Güray Ü, Kısacık HF. Coronary artery perforation during diagnostic coronary angiography resulting in acute pericarditis. Postepy Kardiol Interwencyjnej 2013;9(3):298-300. 